\title{
Avaliação clínica e evolutiva de crianças em programa de atendimento ao uso de fórmulas para alergia à proteína do leite de vaca
}

\author{
Clinical and follow up assessment of children in a program directed at the use of formulas for cow's milk \\ protein allergy
}

Ana Laissa O. Aguiar ${ }^{1}$, Clarissa Marques Maranhão ${ }^{1}$, Lívia Carvalho Spinelli', Roberta Marinho de Figueiredo ${ }^{1}$, Jussara Melo C. Maia², Rosane Costa Gomes ${ }^{3}$, Hélcio de Sousa Maranhão ${ }^{4}$

\section{RESUMO}

Objetivo: Determinar características clínicas e evolutivas de crianças acompanhadas em programa de referência para fornecimento de fórmulas especiais para alergia ao leite de vaca.

Métodos: Estudo descritivo, realizado em amostra de conveniência, com 214 crianças até três anos, com diagnóstico clínico e/ou teste padronizado de provocação oral aberto, referenciadas ao Programa de Fórmulas para Alergia ao Leite de Vaca, em Hospital Universitário Pediátrico de Natal, Rio Grande do Norte (2007/2009). Avaliaram-se dados clínico-epidemiológicos e indicação de fórmulas (soja, hidrolisado ou aminoácido) à consulta inicial, além de resposta clínica e evolução nutricional (Anthro-OMS 2006) após três meses. Aplicaram-se os testes do qui-quadrado e $t$ pareado nas análises, considerando-se significante $p<0,05$.

Resultados: Ao primeiro atendimento, a média de idade foi de 9,0 $\pm 6,9$ meses. Manifestações digestórias foram observadas em $81,8 \%$; cutâneas, em 36,9\%; e respiratórias, em 23,8\%. Escore Z do IMC <-2,0 desvios padrão (DP) foi encontrado em $17,9 \%$ das crianças com sintomas digestórios isolados, em $41,7 \%$ em uso de leite de vaca e em $8,7 \%$ com outras fórmulas $(p<0,01)$. Fórmula de proteína isolada de soja foi usada em $61,2 \%$; hidrolisados, em 35,4\%; e aminoácidos, em 3,3\%. As médias de escore $\mathrm{Z}$ do IMC ao atendimento inicial e após três meses

Instituição: Departamento de Pediatria e Hospital de Pediatria da Universidade Federal do Rio Grande do Norte (UFRN), Natal, RN, Brasil

'Bolsista de Iniciação Científica da Faculdade de Medicina da UFRN, Natal, RN, Brasil

Doutora em Ciências da Saúde pelo Programa de Pós-graduação em Ciências da Saúde da UFRN; Professora Adjunta do Departamento de Pediatria da UFRN, Natal, RN, Brasil

3Pós-Graduanda de Doutorado do Programa de Pós-graduação em Ciências da Saúde da UFRN; Professora - Assistente do Departamento de Pediatria da UFRN, Natal, RN, Brasil

${ }^{4}$ Doutor em Ciências pelo Programa de Pós-graduação em Pediatria da Universidade Federal de São Paulo (Unifesp); Professor Associado do Departamento de Pediatria da UFRN, Natal, RN, Brasil foram, respectivamente, $-0,24 \pm 1,47 \mathrm{DP}$ e $0,00 \pm 1,26 \mathrm{DP}$ $(p=0,251)$, quando em uso de soja, e $-0,70 \pm 1,51 \mathrm{DP}$ e $-0,14 \pm 1,36 \mathrm{DP}(p=0,322)$, em uso de hidrolisado.

Conclusões: Manifestações digestórias da alergia ao leite de vaca foram preponderantes e determinaram maior comprometimento nutricional. As fórmulas de substituição ao leite de vaca mais utilizadas foram de proteína isolada de soja e hidrolisados proteicos. O uso de ambas foi importante para a manutenção do estado nutricional.

Palavras-chave: hipersensibilidade a leite; dietoterapia; substitutos do leite; programas; leite de soja; criança.

\section{ABSTRACT}

Objective: To determine clinical and follow up characteristics of children enrolled in a program to supply formulas for cow's milk allergy.

Methods: descriptive study of a convenience sample composed of 214 children up to three years old, with clinical diagnosis of cow's milk allergy and/or standardized oral challenge, referred to the Program of Formulas for Cow's Milk Allergy at a Pediatric University Hospital, in Natal, Rio Grande do Norte, Brazil (2007/2009). Clinical-epidemiological data and formula indication (soy, protein hydrolysates or aminoacid formula) were assessed at the first consultation. Clinical response and nutritional

Endereço para correspondência:

Hélcio de Sousa Maranhão

Avenida Amintas Barros, 3.673, Bloco I, apto. 502 - Lagoa Nova

CEP 59075-250 - Natal/RN

E-mail: hmaranhao@ufrnet.br

Conflito de interesse: nada a declarar

Recebido em: 26/7/2012

Aprovado em: 15/1/2013 
evolution (Anthro-OMS2006) were observed after three months. Chi-square and paired t-test were used, being $p<0.05$ significant.

Results: At the first consultation, mean age was 9.0 16.9 months. Digestive manifestations occurred in $81.8 \%$; cutaneous ones, in $36.9 \%$ and respiratory ones in $23.8 \%$. BMI Zscore $<-2.0$ standard deviations (SD) was found in $17.9 \%$ of children with isolated digestive symptoms, in $41.7 \%$ of those using cow's milk and in $8.7 \%$ of those using other formulas $(p<0.01)$. The following formulas were used: soy in $61.2 \%$, protein hydrolysates in $35.4 \%$ and aminoacids in $3.3 \%$. Mean BMI Z-scores at initial consultation and after three months were, respectively: $-0.24 \pm 1.47 \mathrm{SD}$ and $0.00 \pm 1.26 \mathrm{SD}$ $(\mathrm{p}=0.251)$, with soy formula, and $-0.70 \pm 1.51 \mathrm{SD}$ and $-0.14 \pm 1.36 \mathrm{SD}(\mathrm{p}=0.322)$ with protein hydrolysates formula.

Conclusions: Digestive manifestations of cow's milk allergy were preponderant, and lead to greater nutritional impairment. The use of replacement formulas (isolated soy protein and protein hydrolysates) was important to maintain the nutritional status.

Key-words: milk hypersensitivity; diet therapy; milk substitutes; programs; soy milk; child.

\section{Introdução}

A proteína do leite de vaca (LV) apresenta potencial alergênico alto e é considerada o mais frequente componente dietético causador de alergia alimentar ${ }^{(1)}$. O diagnóstico da alergia à proteína do leite de vaca (APLV) baseia-se nas manifestações clínicas, na resposta à dieta de exclusão e posterior teste de desencadeamento duplo-cego controlado por placebo (padrão-ouro) ${ }^{(2)}$. No entanto, devido às suas dificuldades práticas, rotineiramente faz-se a opção pelo teste de provocação aberto $^{(3-5)}$. Após a confirmação, recomendam-se novos testes a cada seis a 12 meses, pela possibilidade do desenvolvimento de tolerância, principalmente nos primeiros três anos de vida. Dessa forma, evita-se a exclusão do alimento por um tempo maior que o necessário ${ }^{(4)}$. Seu tratamento consiste na retirada do LV e seus derivados da dieta e na sua substituição por fórmulas à base de proteína isolada de soja, de proteínas extensamente hidrolisadas ou de aminoácidos, a depender de critérios clínicos ${ }^{(6)}$.

Dados concernentes à prevalência de APLV são escassos, e o diagnóstico é de difícil confirmação, uma vez que as manifestações clínicas são variadas - comprometendo sistema digestório, respiratório e pele — e podem ser confundidas com outras hipersensibilidades alimentares. Além disso, os testes de provocação para o diagnóstico ainda são pouco utilizados na prática clínica.

Em virtude do alto custo das fórmulas hipoalergênicas utilizadas em substituição ao LV, implantaram-se no país, nos últimos anos, por meio de iniciativas isoladas, programas governamentais, sejam municipais ou estaduais, para avaliar a indicação de tais fórmulas, com o objetivo de minimizar seu uso desnecessário e reduzir os recursos financeiros utilizados, assim como os riscos nutricionais advindos de uma indicação inadequada.

A escassez de publicações no país que apresentam análise de casuísticas advindas desses programas motivou a realização deste estudo, que pode trazer contribuição para a implantação e/ou gerenciamento de outros programas semelhantes, em distintas regiões, e consequente adoção de estratégias para o manejo e tratamento da APLV. Dessa forma, o objetivo do presente trabalho foi determinar as características clínico-epidemiológicas, dietéticas e evolutivas dos pacientes encaminhados ao Programa de Avaliação da Indicação e Uso de Fórmulas Infantis Especiais para Alergia à Proteína do Leite de Vaca (PAIUFA), implantado em 2007 no Hospital de Pediatria da Universidade Federal do Rio Grande do Norte (Hosped-UFRN), serviço de referência para o Estado.

\section{Método}

Trata-se de estudo descritivo, desenvolvido no HospedUFRN, no período de janeiro de 2007 a dezembro de 2009. A amostra de conveniência foi constituída por 214 crianças, na faixa etária de zero a três anos, residentes no Estado do Rio Grande do Norte, atendidas nas unidades do Sistema Único de Saúde (SUS) ou na rede privada, referenciadas por médicos ou nutricionistas ao PAIUFA por apresentarem sintomas atribuídos à ingestão de proteína de LV.

Avaliaram-se todas as crianças atendidas no período do estudo e que receberam parecer favorável de pelo menos um dos três gastroenterologistas pediátricos do Programa para a obtenção de fórmulas para APLV, sendo preenchido questionário específico, com ou sem realização de teste de provocação oral (TPO) aberto no serviço. Considerou-se o teste positivo com o reaparecimento de sinais e sintomas imediatos ou tardios compatíveis com APLV, após a reintrodução do LV no cardápio da criança. Para aquelas que não foram submetidas ao TPO, consideraram-se as evidências clínicas após a introdução do LV e a resolução dos sinais e sintomas com a dieta de eliminação ${ }^{(4,5)}$. 
O TPO não foi realizado em casos de reações prévias imediatas graves (caraterizadas por vômitos e sibilância importantes, dificuldades respiratórias, urticária grave, angioedema, anafilaxia e síndrome da enterocolite induzida por proteína alimentar, ocorridas em minutos até 2 horas após a exposição ao LV), recusa peremptória dos familiares, crianças com diagnóstico nutricional de magreza e magreza acentuada ou naquelas com procedimento já realizado e observado pelo pediatra acompanhante.

No questionário, registraram-se dados epidemiológicos e clínicos do primeiro atendimento, como idade, sexo, procedência, referência (SUS ou consultório privado), antecedentes gestacionais, familiares e alimentares, sintomatologia apresentada e tipo de fórmula indicada.

No acompanhamento evolutivo, observaram-se a resposta clínica dos pacientes, a conduta dietética (manutenção, substituição ou suspensão da fórmula) e a evolução do estado nutricional, avaliando-se tais parâmetros três meses após a primeira consulta. Utilizaram-se os padrões de referência da OMS-2006 para avaliar o estado nutricional, a partir da análise do escore $\mathrm{Z}$ do índice de massa corpórea (IMC) ${ }^{(7)}$. Para a classificação do estado nutricional, tendo em vista os valores de corte preconizados, reuniram-se as crianças em dois grupos, a saber: escore $Z$ do IMC $<-2,0$ desvio padrão (DP) e escore $Z$ do IMC $\geq-2,0 D P$. Os dados foram avaliados por meio do programa Anthro-OMS 2006.

Para o processamento dos dados e as análises estatísticas, utilizou-se o software Statistical Package for the Social Sciences (SPSS), versão 17.0. A fim de comparar as variáveis entre grupos distintos, estratificou-se a amostra pela faixa etária ( $<6, \geq 6$ e $<12, \geq 12$ meses), sexo, estado nutricional (escore $\mathrm{Z}$ do IMC $<-2,0 \mathrm{DP}=$ magreza ou magreza acentuada, escore $\mathrm{Z}$ do IMC $\geq-2,0 \mathrm{DP}=$ eutrofia, sobrepeso ou obesidade), manifestações clínicas (cutâneas, respiratórias ou digestórias, isoladas ou associadas) e tipo de fórmula utilizada (à base de proteína isolada de soja, hidrolisado proteico ou fórmula de aminoácido). Utilizou-se o teste do qui-quadrado ou exato de Fisher para comparar proporções, e o teste $t$ pareado a fim de comparar o estado nutricional dos indivíduos antes e depois da intervenção dietética. Usou-se o Teste de Kolmogorov-Smirnov para confirmação da normalidade dessa variável. Admitiu-se nível de significância de $5 \%(p<0,05)$.

O estudo foi aprovado pelo Comitê de Ética em Pesquisa do Hospital Universitário Onofre Lopes da Universidade Federal do Rio Grande do Norte (UFRN). Todos os pais ou responsáveis assinaram o termo de consentimento livre e esclarecido.

\section{Resultados}

Avaliaram-se 214 crianças com idade média de 9,0 $( \pm 6,9)$ meses ao primeiro atendimento, sendo $58,4 \%$ do sexo masculino e $41,6 \%$ do feminino; $68,1 \%$ procedentes da cidade do Natal e 31,9\% de demais cidades do Estado; $59,4 \%$ provenientes de serviço privado de saúde e 40,6\% de serviço público.

A idade média de início dos sintomas foi de 2,8 2 2,5 me-

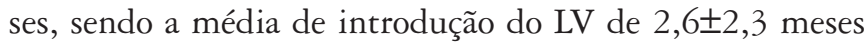
e mediana de 2,0 meses. Embora acometidas por sinais e sintomas envolvendo simultaneamente distintos sistemas, manifestações isoladas do sistema digestório, da pele e do trato respiratório foram encontradas em 49,5, 11,2 e 2,3\%, com idade média de início de sintomas de 2,0土1,9 meses, $3,3 \pm 2,3$ meses e 4,0 $\pm 3,0$ meses, respectivamente. A Figura 1 discrimina os sintomas mais frequentemente encontrados e os percentuais gerais de acometimento dos tratos respiratório e digestório e da pele.

A avaliação do estado nutricional pelo escore $Z$ do IMC evidenciou magreza ou magreza acentuada em $12,9 \%$, eutrofia em $67,8 \%$, risco de sobrepeso em $15,2 \%$ e sobrepeso ou obesidade em $4,1 \%$.

Ao chegarem ao serviço, os percentuais de crianças em uso de LV, fórmula à base de soja, hidrolisado proteico ou de aminoácidos eram 10,5, 65,1, 19,8 e 4,7\%, respectivamente. Daquelas que faziam uso de LV $(n=12)$, encontraram-se magreza (escore $Z$ do IMC $<-2,0 \mathrm{DP}$ ) ou magreza acentuada (escore $Z$ do IMC <-3,0DP) em 41,7\% ( $n=5)$ e, daquelas em uso de outras fórmulas ( $\mathrm{n}=126)$, em 8,7\% ( $\mathrm{n}=11)(p=0,001)$. Crianças com manifestações isoladas do sistema digestório apresentaram magreza ou magreza acentuada em 17,9\%, o que não se encontrou em nenhuma criança com acometimento isolado do sistema respiratório ou da pele.

As fórmulas indicadas ou mantidas ao primeiro atendimento no PAIUFA, independentemente da idade da criança, eram à base de proteína isolada de soja em $61,2 \%$ dos casos, hidrolisado proteico em $35,4 \%$ e de aminoácidos em 3,3\%. A Tabela 1 demonstra as fórmulas prescritas de acordo com a faixa etária da casuística. A frequência dos sintomas distribuídos de acordo com a faixa etária é apresentada na Tabela 2.

Para os indivíduos que apresentaram sintomas exclusivamente digestórios, prescreveram-se fórmulas à base de proteína isolada de soja em $51 \%$, de hidrolisado proteico em 43,3\% e de aminoácidos em 5,8\%. Para aqueles com sintomas exclusivamente cutâneos, prescreveram-se fórmulas 
à base de proteína isolada de soja em 78,3\% e hidrolisado proteico em $21,7 \%$, não se observando prescrição de fórmulas de aminoácidos. Todas as crianças com sintomas exclusivamente respiratórios receberam prescrição de fórmula à base de proteína isolada de soja.

Dos 214 pacientes, $63(29,4 \%)$ realizaram TPO em algum momento do acompanhamento médico. Destes, $59(93,6 \%)$ apresentaram resposta positiva nas primeiras quatro semanas após provocação, dois $(3,2 \%)$ na quinta semana, manifestada pela recidiva das manifestações clínicas, e dois não retornaram ao seguimento depois do primeiro mês de observação após o TPO. Dentre os 151 pacientes que não realizaram o TPO, os motivos mais prevalentes foram: história de provocação anterior bem estabelecida $(29,8 \%)$, reações prévias imediatas graves $(29,2 \%)$, magreza ou magreza acentuada $(17,2 \%)$, recusa dos familiares em

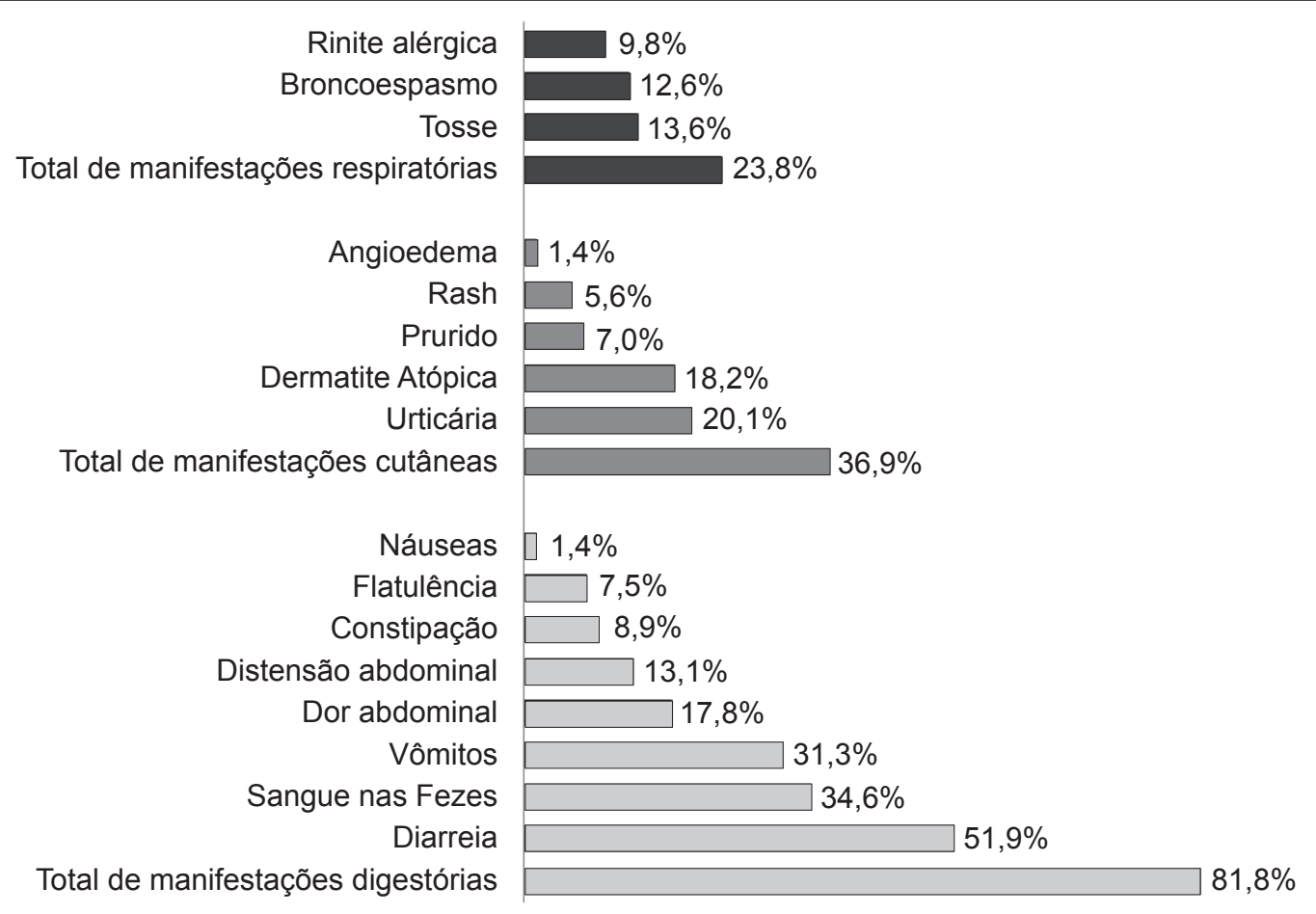

Manisfestações respiratórias

Manisfestações cutâneas

Manisfestações digestórias

Figura 1 - Principais sinais e sintomas nos sistemas acometidos em crianças atendidas no Programa de Avaliação da Indicação e Uso de Fórmulas Infantis Especiais para Alergia à Proteína do Leite de Vaca, do Hospital de Pediatria da Universidade Federal do Rio Grande do Norte, de janeiro de 2007 a dezembro de 2009

Tabela 1 - Indicação de fórmulas infantis especiais ao primeiro atendimento de crianças no Programa de Avaliação da Indicação e Uso de Fórmulas Infantis Especiais para Alergia à Proteína do Leite de Vaca, do Hospital de Pediatria da Universidade Federal do Rio Grande do Norte, de janeiro de 2007 a dezembro de 2009

\begin{tabular}{|c|c|c|c|c|c|c|}
\hline \multirow{3}{*}{$\begin{array}{l}\text { Faixa etária } \\
\text { (meses) }\end{array}$} & \multicolumn{6}{|c|}{ Fórmula indicada } \\
\hline & \multicolumn{2}{|c|}{ Soja } & \multicolumn{2}{|c|}{$\begin{array}{c}\text { Hidrolisado } \\
\text { proteico }\end{array}$} & \multicolumn{2}{|c|}{ Aminoácido } \\
\hline & $\mathbf{n}$ & $\%$ & $\mathbf{n}$ & $\%$ & $\mathbf{n}$ & $\%$ \\
\hline 0 a $6(n=96)$ & 43 & 44,8 & 48 & 50,0 & 5 & 5,2 \\
\hline 6 a $12(n=60)$ & 42 & 70,0 & 17 & 28,3 & 1 & 1,7 \\
\hline$\geq 12(n=58)$ & 46 & 79,3 & 11 & 19,0 & 1 & 1,7 \\
\hline
\end{tabular}

Tabela 2 - Comprometimento digestório, cutâneo e respiratório em crianças atendidas no Programa de Avaliação da Indicação e Uso de Fórmulas Infantis Especiais para Alergia à Proteína do Leite de Vaca, do Hospital de Pediatria da Universidade Federal do Rio Grande do Norte, de janeiro de 2007 a dezembro de 2009, por faixa etária

\begin{tabular}{|c|c|c|c|c|c|c|}
\hline \multirow{3}{*}{$\begin{array}{l}\text { Faixa etária } \\
\text { (meses) }\end{array}$} & \multicolumn{6}{|c|}{ Sintomas } \\
\hline & \multicolumn{2}{|c|}{ Digestórios } & \multicolumn{2}{|c|}{ Cutâneos } & \multicolumn{2}{|c|}{ Respiratórios } \\
\hline & $\mathbf{n}$ & $\%$ & $n$ & $\%$ & $\mathbf{n}$ & $\%$ \\
\hline 0 a $6(n=96)$ & 81 & 84,4 & 25 & 26,0 & 16 & 16,7 \\
\hline 6 a $12(n=60)$ & 49 & 81,7 & 24 & 40,0 & 18 & 30,0 \\
\hline$\geq 12(n=58)$ & 44 & 75,9 & 30 & 51,7 & 17 & 29,3 \\
\hline
\end{tabular}


Tabela 3 - Evolução do estado nutricional de 67 crianças atendidas no Programa de Avaliação da Indicação e Uso de Fórmulas Infantis Especiais para Alergia à Proteína do Leite de Vaca, do Hospital de Pediatria da Universidade Federal do Rio Grande do Norte, de janeiro de 2007 a dezembro de 2009, após três meses de uso de fórmula de soja ou hidrolisado proteico

\begin{tabular}{|c|c|c|c|c|c|c|c|c|}
\hline \multirow{3}{*}{ Fórmula em uso } & \multicolumn{4}{|c|}{ Primeira consulta $^{*}$} & \multicolumn{4}{|c|}{ Após três meses ${ }^{* *}$} \\
\hline & \multicolumn{2}{|c|}{$\begin{array}{c}\text { Escore } Z \text { do IMC - } \\
<-2 D P\end{array}$} & \multicolumn{2}{|c|}{$\begin{array}{c}\text { Escore } Z \text { do IMC - } \\
\quad \geq-2 D P\end{array}$} & \multicolumn{2}{|c|}{$\begin{array}{c}\text { Escore } \mathrm{Z} \text { do IMC - } \\
<-2 \mathrm{DP}\end{array}$} & \multicolumn{2}{|c|}{$\begin{array}{c}\text { Escore } Z \text { do IMC - } \\
\geq-2 D P\end{array}$} \\
\hline & $\mathbf{n}$ & $\%$ & $\mathbf{n}$ & $\%$ & $n$ & $\%$ & $n$ & $\%$ \\
\hline Soja & 5 & 9,6 & 47 & 90,4 & 2 & 3,8 & 50 & 96,2 \\
\hline Hidrolisado proteico & 3 & 20,0 & 12 & 80,0 & 0 & 0,0 & 15 & 100,0 \\
\hline
\end{tabular}

Teste exato de Fisher: " $p=0,385 ;{ }^{* *} p=1,000$; IMC: índice de massa corpórea; DP: desvio padrão

fazer o procedimento $(15,2 \%)$ e não retorno para o acompanhamento no momento agendado $(8,60 \%)$. Algumas crianças congregavam mais de um desses fatores.

Do total da casuística, $62,1 \%$ retornaram para a reavaliação após três meses. Daquelas em uso de fórmula à base de proteína isolada de soja ( $\mathrm{n}=91)$, houve manutenção da prescrição em 83 (91,2\%), substituição em sete $(7,7 \%)$ — todas para hidrolisado proteico — e suspensão em uma $(1,1 \%)$. Quanto às crianças em uso de hidrolisado proteico $(\mathrm{n}=37)$, a fórmula foi mantida em $30(81,1 \%)$, substituída em seis $(16,2 \%)$ - cinco para soja e uma para fórmula de aminoácido - e suspensa em uma $(2,7 \%)$. Para as crianças em uso de aminoácidos $(n=5)$, a fórmula foi mantida em uma, substituída em três — todas para hidrolisado proteico - e suspensa em uma.

A Tabela 3 demonstra a evolução do estado nutricional das crianças, quando avaliadas após três meses de uso de fórmula de soja ou hidrolisado proteico. Em virtude do pequeno número de crianças em uso de fórmula de aminoácido, a análise desses dados tornou-se prejudicada. As médias de escore $\mathrm{Z}$ do IMC das crianças em uso de fórmula de soja na primeira consulta e após três meses foram $-0,24 \pm 1,47 \mathrm{DP}$ e $0,00 \pm 0,26 \mathrm{DP}$, respectivamente $(\phi=0,251)$. Para aquelas em uso de hidrolisado proteico, as médias foram $-0,70 \pm 1,51 \mathrm{DP}$ e $-0,14 \pm 1,36 \mathrm{DP}(p=0,322)$.

\section{Discussão}

O PAIUFA é a referência atual no Estado do Rio Grande do Norte para crianças com o diagnóstico clínico suspeito ou comprovado de alergia alimentar, com ênfase em APLV. A partir de sua criação, houve um incremento acentuado dessa demanda ao Hosped-UFRN. Nesse sentido, torna-se importante o conhecimento das características epidemiológicas e clínicas da clientela atendida, trazendo subsídios para novas ações diagnósticas, intervencionistas e preventivas.
Atentando-se à idade média ao primeiro atendimento no PAIUFA e às idades de início dos sintomas sugestivos de APLV, observa-se um intervalo aproximado de seis meses. Tal fato pode se relacionar a dificuldades diagnósticas anteriores, procura ou encaminhamento tardio ao serviço ou uso prévio de fórmulas especiais prescritas pelos profissionais acompanhantes e adquiridas por meio dos recursos da família.

A mediana de introdução do LV (2,0 meses) reflete a presença altamente precoce desse alérgeno no cardápio da criança, em um momento totalmente compatível com o aleitamento materno exclusivo ${ }^{(8)}$ - condição de alto risco para o surgimento da $\mathrm{APLV}^{(9)}$. Em uso exclusivo de leite materno, essa exposição poderia ser postergada para um momento mais apropriado.

A prevalência das manifestações clínicas da APLV depende da casuística de distintos serviços ${ }^{(10,11)}$, como a pneumoalergologia, a gastroenterologia e a dermatologia. No entanto, a predominância dos sintomas digestórios no PAIUFA ressalta a importância do comprometimento digestivo, assim como em outros estudos ${ }^{(12,13)}$, visto que o Programa é uma referência disponível a todos os profissionais e especialidades supostamente envolvidos com a APLV.

Dentre as manifestações sugestivas de APLV que motivaram o encaminhamento ao Programa, diarreia foi a manifestação digestória mais prevalente $(51,9 \%)$; porém, constipação intestinal foi encontrada em $8,9 \%$, o que chama atenção para essa forma recentemente reconhecida de apresentação da $\mathrm{APLV}^{(14,15)}$. Colite alérgica, evidenciada pela presença de sangue nas fezes e envolvida entre os fenômenos da $\mathrm{APLV}^{(16)}$, foi encontrada em 34,6\%. Apesar da predominância de urticária $(20,1 \%)$ nos participantes deste estudo, há relevância crescente dos quadros de dermatite atópica (DA) como manifestação de alergia alimentar ${ }^{(17)}$, o que se encontrou em $18,2 \%$. 
Ao se compararem as crianças com acometimento exclusivo dos sistemas digestório, respiratório ou da pele em relação ao seu estado nutricional, encontrou-se magreza exclusivamente em indivíduos com comprometimento do primeiro, o que pode refletir a participação do componente disabsortivo nesse processo. Torna-se interessante ressaltar que as crianças que chegaram ao serviço, ainda em uso de LV, apresentavam maiores percentuais de magreza ou magreza acentuada do que aquelas que já não mais usavam tal componente em seus cardápios, substituindo-o por outras fórmulas.

Das crianças avaliadas no Programa, $83,1 \%$ já estavam em uso de fórmulas especiais, à base de soja, hidrolisado proteico ou aminoácido, no primeiro atendimento. Em torno de $50 \%$ realizaram TPO para confirmação da APLV, seja antes (observado pelo médico acompanhante) ou após a chegada ao serviço (durante o acompanhamento).

Apesar da recomendação do TPO para o diagnóstico clínico de APLV, esse procedimento apresenta várias objeções na prática clínica diária, sendo realizado aquém do desejável. O percentual de crianças que realizaram o teste traduz a dificuldade do pediatra em indicá-lo. As crianças com APLV submetidas a dieta de eliminação e uso de fórmulas adequadas, quando desnutridas, passam por um processo de aumento ponderal (e, posteriormente, de estatura). Interrompê-lo para uma provocação precoce, em poucas semanas, com finalidade estritamente diagnóstica, talvez seja discutível, em virtude de trazê-las aos patamares anteriores, com novo prejuízo para seu crescimento e desenvolvimento. Da mesma forma, há situações clínicas em que se desconsidera a obrigatoriedade do TPO, como naquelas com manifestações mais imediatas, sobretudo as mediadas por IgE, conforme recente publicação de Koletzko et al ${ }^{(18)}$. Expor os pacientes a novos e sérios riscos pode ser dispensável. Vale ressaltar que o PAIUFA não tem como condição sine qua non, para o recebimento das fórmulas especiais, a realização do TPO no serviço, apesar de recomendá-lo a todo médico assistente e de fazê-lo quando a situação é adequada. Porém, a maioria dos médicos não dispõe de estrutura física para execução segura do procedimento. Devem-se considerar, ainda, as questões éticas, legais e profissionais ao se realizar o TPO em pacientes encaminhados aos programas, mas que, na verdade, são acompanhados por seus médicos de origem e chegam ao serviço com o diagnóstico já estabelecido, a fim de receberem as fórmulas de alto custo. Assumir tal responsabilidade é duplamente comprometedor pelo risco de se produzirem reações inesperadas e não tratar o paciente, com implicações ainda obscuras. Ademais, há familiares que recusam veementemente o procedimento, tendo em vista que seus filhos encontram-se muito bem sob a dieta. Essas são considerações de difícil solução e pouco debatidas. Por todos os motivos aventados, admite-se que uma parcela de crianças pode permanecer em dieta de exclusão sem uma prova definitiva da APLV. Portanto, a rigidez da principal ferramenta diagnóstica, seja o TPO aberto, cego ou duplo-cego, torna-o subutilizado na prática e passível de discussão, apesar da extrema valorização de seus preceitos teóricos.

Os percentuais de prescrição ou manutenção das fórmulas à primeira consulta certamente sofreram influência da prescrição prévia pelos profissionais responsáveis pelo encaminhamento, chamando atenção à vasta utilização das fórmulas à base de proteína isolada de soja ${ }^{(11,19)}$, apesar de o algoritmo do PAIUFA não indicar a prescrição dessas fórmulas em menores de seis meses, além das evidências de seu potencial alergênico e das controvérsias quanto à estimulação estrogênica e carcinogênese ${ }^{(20-23)}$. Seu uso foi maior em crianças acima de seis meses. Por outro lado, maiores percentuais de prescrição de hidrolisado proteico e de fórmulas de aminoácidos ocorreram para crianças menores de seis meses, nas quais predominaram as manifestações digestórias, quando comparadas às cutâneas e respiratórias ${ }^{(6)}$.

A baixa ocorrência de efeitos adversos ao uso da soja nesta casuística traz questionamentos acerca de condições particulares que possam promover maior tolerância à sua prescrição. O seguimento e a observação estreita desses indivíduos em relação a seu estado nutricional e a manifestações de intolerância ou outras por maior período, condição essa em andamento, permitirão melhor esclarecimento desses achados, quando comparadas as crianças em uso das distintas fórmulas. Porém, as novas diretrizes da European Society for Paediatric Gastroenterology, Hepatology and Nutrition (ESPGHAN), de 2012, são mais flexíveis quanto à utilização da proteína isolada de soja após o primeiro semestre de vida, não só nas formas de APLV mediadas por IgE, mas também em manifestações do sistema digestório, como alternativa ao hidrolisado proteico, ainda considerado a primeira opção. Os autores apontam baixos percentuais de intolerância à soja (10 a 14\%), em detrimento de estatísticas anteriores, estando seu uso indicado diante de má aceitação aos hidrolisados, dificuldades na sua aquisição por motivos de custos ou preferências familiares, por exemplo, veganos ${ }^{(18)}$.

É preciso levar em consideração o alto custo das fórmulas para a intervenção, devendo-se priorizar sua eficácia, segurança, relação custo-benefício e indicação nas distintas faixas de idade. Os programas governamentais 
de aquisição e fornecimento de fórmulas especiais para APLV, a serem implantados ou em andamento, assim como os serviços de saúde que atendem essa clientela, devem conhecer o perfil de seu público-alvo ou de casuísticas similares que possam auxiliar no planejamento de ações clínicas, intervenções dietéticas e aplicação de recursos de forma adequada e equilibrada.

A evidência do início precoce dos sintomas de APLV reforça a relevância do incentivo ao aleitamento materno exclusivo nos primeiros seis meses de vida e sua manutenção até os dois anos, como principal estratégia de prevenção.

\section{Referências bibliográficas}

1. Joneja JM. Infant food allergy: where are we now? J Parenter Enteral Nutr 2012;36 (1 Suppl):49S-55S.

2. Baehler P, Chad Z, Gurbindo C, Bonin AP, Bouthillier L, Seidman EG. Distinct patterns of cow's milk allergy in infancy defined by prolonged, two-stage doubleblind, placebo-controlled food challenges. Clin Exp Allergy 1996;26:254-61.

3. Niggemann B, Sielaff B, Beyer K, Binder C, Wahn U. Outcome of double-blind, placebo-controlled food challenge tests in 107 children with atopic dermatitis. Clin Exp Allergy 1999;29:91-6.

4. Toporovski MS, Vieira MC, Spolidoro JV, Morais MB, Fagundes-Neto U. Alergia ao Leite de Vaca. In: Lopez FA, Campos-Junior D, editors. Tratado de Pediatria. São Paulo: Manole; 2007. p.863-71.

5. Lins MD, Horowitz MR, Silva GA, Motta ME. Oral food challenge test to confirm the diagnosis of cow's milk allergy. J Pediatr (Rio J) 2010;86:285-9.

6. Kemp AS, Hill DJ, Allen KJ, Anderson K, Davidson GP, Day AS et al. Guidelines for the use of infant formulas to treat cows milk protein allergy: an Australian consensus panel opinion. Med J Aust 2008;188:109-12.

7. Brasil. Ministério da Saúde. Política Nacional de Alimentação e Nutrição [homepage on the Internet]. Curvas de crescimento da Organização Mundial da Saúde - OMS [cited 2010 Mar 31]. Available from:

8. http://nutricao.saude.gov.br/sisvan.php?conteudo=curvas_cresc_oms

9. World Health Organization. The optimal duration of exclusive breastfeeding: report of an expert consultation. Geneva: WHO; 2001.

10. Greer FR, Sicherer SH, Burks AW; American Academy of Pediatrics Committee on Nutrition; American Academy of Pediatrics Section on Allergy and Immunology.. Effects of early nutritional interventions on the development of atopic disease in infants and children: the role of maternal dietary restriction, breastfeeding, timing of introduction of complementary foods, and hydrolyzed formulas. Pediatrics 2008;121:183-91.

11. Husby S. Food allergy as seen by a paediatric gastroenterologist. J Pediatr Gastroenterol Nutr 2008;47 (Suppl 2):S49-52.

12. Ngamphaiboon J, Chatchatee P, Thongkaew T. Cow's milk allergy in Thai children. Asian Pac J Allergy Immunol 2008;26:199-204.

13. Sicherer SH. Clinical aspects of gastrointestinal food allergy in childhood. Pediatrics 2003;111:1609-16.
Apesar do comprometimento cutâneo e respiratório na APLV, as manifestações do sistema digestório ganham primordial importância devido à sua grande frequência e às repercussões desfavoráveis sobre o estado nutricional. O uso de hidrolisados proteicos é prioritário como dieta de substituição para crianças com APLV, porém as fórmulas de proteína isolada de soja ainda constituem alternativa favorável, sobretudo para crianças acima de seis meses. As fórmulas de aminoácidos devem ser restritas aos casos de intolerância aos hidrolisados, alergias múltiplas ou quadros clínicos graves, com comprometimento nutricional intenso.
14. Vieira MC, Morais MB, Spolidoro JV, Toporovski MS, Cardoso AL, Araujo GT et al. A survey on clinical presentation and nutritional status of infants with suspected cow' milk allergy. BMC Pediatrics 2010;10:25.

15. Allen KJ, Davidson GP, Day AS, Hill DJ, KempAS, Peake JE et al. Management of cow's milk protein allergy in infants and young children: an expert panel perspective. J Paediatr Child Health 2009;45:481-6.

16. El-Hodhod MA, Younis NT, Zaitoun YA, Daoud SD. Cow's milk allergy related pediatric constipation: appropriate time of milk tolerance. Pediatr Allergy Immunol 2010;21:e407-12.

17. Poddar U, Yachha SK, Krishnani N, Srivastava A. Cow's milk protein allergy: an entity for recognition in developing countries. J Gastroenterol Hepatol 2010;25:178-82.

18. Sicherer SH, Sampson HA. Food hypersensitivity and atopic dermatitis: pathophysiology, epidemiology, diagnosis, and management. J Allergy Clin Immunol 1999;104:S114-22.

19. Koletzko S, Niggemann B, Arato A, Dias JA, Heuschkel R, Husby S, Mearin ML, Papadopoulou A, Ruemmele FM, Staiano A, Schäppi MG, Vandenplas Y; European Society of Pediatric Gastroenterology, Hepatology, and Nutrition. Diagnostic approach and management of cow's-milk protein allergy in infants and children: ESPGHAN GI Committee practical guidelines. J Pediatr Gastroenterol Nutr 2012;55:221-9.

20. Sladkevicius E, Nagy E, Lack G, Guest JF. Resource implications and budget impact of managing cow milk allergy in the UK. J Med Econ 2010;13:119-28.

21. Vandenplas $Y$, Brueton $M$, Dupont $C$, Hill D, Isolauri E, Koletzko $S$ et al. Guidelines for the diagnosis and management of cow's milk protein allergy in infants. Arch Dis Child 2007;92:902-8.

22. Gilchrist JM, Moore MB, Andres A, Estroff JA, Badger TM. Ultrasonographic patterns of reproductive organs in infants fed soy formula: comparisons to infants fed breast milk and milk formula. J Pediatr 2010;156:215-20.

23. Cao Y, Calafat AM, Doerge DR, Umbach DM, Bernbaum JC, Twaddle NC et al. Isoflavones in urine, saliva, and blood of infants: data from a pilot study on the estrogenic activity of soy formula. J Expo Sci Environ Epidemiol 2009;19:223-34.

24. Turck D. Soy protein for infant feeding: what do we know? Curr Opin Clin Nutr Metab Care 2007;10:360-5. 